\title{
Noise-induced phase separation: Mean-field results
}

\author{
M. Ibañes, ${ }^{1}$ J. García-Ojalvo, ${ }^{2,3}$ R. Toral, ${ }^{4}$ and J. M. Sancho ${ }^{1}$ \\ ${ }^{1}$ Departament d'Estructura i Constituents de la Matèria, Universitat de Barcelona, Diagonal 647, E-08028 Barcelona, Spain \\ ${ }^{2}$ Departament de Física i Enginyeria Nuclear, Universitat Politècnica de Catalunya, Colom 11, E-08222 Terrassa, Spain \\ ${ }^{3}$ Institut für Physik, Humboldt Universität zu Berlin, Invalidenstr. 110, D-10115 Berlin, Germany \\ ${ }^{4}$ Instituto Mediterráneo de Estudios Avanzados (IMEDEA CSIC-UIB) and Departament de Física, Universitat de les Illes Balears, \\ E-07071 Palma de Mallorca, Spain
}

(Received 9 March 1999)

\begin{abstract}
We present a study of a phase-separation process induced by the presence of spatially correlated multiplicative noise. We develop a mean-field approach suitable for conserved-order-parameter systems and use it to obtain the phase diagram of the model. Mean-field results are compared with numerical simulations of the complete model in two dimensions. Additionally, a comparison between the noise-driven dynamics of conserved and nonconserved systems is made at the level of the mean-field approximation.
\end{abstract}

[S1063-651X(99)01410-5]

PACS number(s): 05.40.-a, 64.60.-i

\section{INTRODUCTION}

Many theoretical and experimental observations confirm nowadays the existence of noise-induced order. Phenomena such as noise-induced transitions [1], stochastic resonance [2], and noise-induced transport [3] are examples of the ordering features of fluctuations in purely temporal dynamical systems. Additionally, recent years have witnessed an increasing interest in noise-induced phenomena in spatially extended systems (see [4] for a recent review). Some of the topics studied in this respect include noise-induced patterns $[5,6]$, noise-induced phase transitions [7-9], spatiotemporal stochastic resonance $[10,11]$, noise-induced fronts [12], noise-supported traveling structures in excitable media [13], and noise-sustained convective structures $[14,15]$. We are concerned in this paper with the phenomenon of noiseinduced phase separation, recently observed in systems with conserved dynamics [16].

Several analytical methods have been used so far [4] to examine the above-mentioned spatiotemporal problems. By way of example, the stability of a homogeneous state with respect to small perturbations of arbitrary wave number can be analyzed in a linear approximation. Such a linear stability analysis shows that pattern-forming transitions are nontrivially affected by multiplicative noise [5,17]. From a more fundamental point of view, systems exhibiting phase transitions in a statistical-mechanics sense can be investigated by means of the dynamic renormalization group $[18,19]$, which shows that under certain conditions a new genuine nonequilibrium universality class arises due to the presence of multiplicative noise [20,21]. A third fruitful approach is based on the well-known mean-field approximation, widely used in the context of equilibrium statistical mechanics, and that has been recently extended to nonequilibrium systems under the influence of external noise $[7,22]$. In this context, the approximation is introduced by assuming that the interaction between a certain spatial point and its neighbors occurs through a mean value of the field, which corresponds to its statistical average at the given point. This approach has led to the prediction of noise-induced ordering and disordering phase transitions (NIOTs and NIDTs), which has been successfully verified (at least qualitatively) by numerical simulations in different models [7,8,21,23]. The advantages of this procedure as compared to, e.g., linear-stability approaches lie in its ability to describe the system arbitrarily far from the transition point and to take into account the influence of spatial coupling strength, which arises naturally in discretized systems. In this way, the mean-field analysis can successfully explain the existence of successive NIOTs and NIDTs (also called reentrant transitions in this context) as a single control parameter is varied.

The aim of this paper is to perform a somewhat detailed study, using the mean-field approximation technique, of the phenomenon of noise-induced phase separation. This phenomenon has been recently predicted by a linear stability approach and confirmed by numerical simulations $[4,16]$. It arises in spatiotemporal systems whose dynamics is conserved, in the sense that the spatial average of the field does not vary with time, but depends only on the initial conditions of the system. Due to this fact, a standard mean-field approach cannot be applied in this case, because no change in the mean field will be observed as a given control parameter is varied (and hence no phase transition can be found in this way). Therefore, an extension of the procedure is needed in order to handle this situation. The present work is devoted to developing such an extension, and applying the results to the particular case of noise-induced phase separation mentioned above. The outline of the rest of the paper is the following. Section II introduces the general system that will be investigated, along with the particular model to which the obtained results will be applied. A comparison between conserved and nonconserved dynamics is also briefly sketched. Section III reviews the mean-field procedure for nonconserved systems, and extends it to include the effect of spatial correlation of the external noise. Section IV introduces the generalized mean-field approach for conserved systems. Section V discusses the limit of strong spatial coupling of the procedure, and compares the corresponding results with those coming from linear stability analysis. Throughout all these sections, a comparison with respective numerical simulations of the 
complete model is made. Finally, some conclusions are stated in Sec. VI.

\section{CONSERVED AND NONCONSERVED DYNAMICAL MODELS}

The spatiotemporal dynamics of a nonequilibrium system in the presence of both internal and external noise can be described by the following Langevin equation [24] for the time evolution of the $d$-dimensional scalar field $\phi(\vec{x}, t)$ :

$$
\frac{\partial \phi(\vec{x}, t)}{\partial t}=-\Gamma\left[\frac{\delta F}{\delta \phi}+g(\phi) \xi(\vec{x}, t)\right]+\eta(\vec{x}, t),
$$

where the additive noise $\eta(\vec{x}, t)$ is Gaussian and white, with zero mean and correlation

$$
\left\langle\eta(\vec{x}, t) \eta\left(\vec{x}^{\prime}, t^{\prime}\right)\right\rangle=2 \varepsilon \Gamma \delta\left(\vec{x}-\vec{x}^{\prime}\right) \delta\left(t-t^{\prime}\right) .
$$

The intensity of the noise is measured by the parameter $\varepsilon$. The existence of the factor $\Gamma$ in correlation (2) is a sign of the internal character of this noise, in whose only presence $(g=0)$ the system can exhibit equilibrium properties. The multiplicative noise term $\xi(\vec{x}, t)$, on the other hand, is external and brings the system out of equilibrium. It may arise, for instance, from a fluctuating control parameter. It is also Gaussian with zero mean, but its correlation will be assumed in principle to have a nontrivial structure in space:

$$
\left\langle\xi(\vec{x}, t) \xi\left(\vec{x}^{\prime}, t^{\prime}\right)\right\rangle=2 \sigma^{2} c\left(\left|\vec{x}-\vec{x}^{\prime}\right|\right) \delta\left(t-t^{\prime}\right),
$$

where $c\left(\left|\vec{x}-\vec{x}^{\prime}\right|\right)$ is the spatial correlation function of the external noise and $\sigma^{2}$ is its intensity.

Different and physically motivated choices for $\Gamma$ will lead to a variety of dynamical and steady-state phenomenologies. The particular case of $\Gamma=-\nabla^{2}$ (called model $B$ in the literature of critical phenomena) is appropriate to describe a system in which the global quantity $\int \phi(\vec{x}, t) d^{d} \vec{x}$ is conserved in time. Physical realizations of this system include the case of phase separation in binary alloys. In this case, an initial mixture of the two components may undergo, for some values of the control parameters, a separation process which, depending on the initial relative concentrations of each component, takes the form of spinodal decomposition or nucleation [25]. In this paper we will be mainly concerned with the conserved case, although a comparison will also be made with the corresponding nonconserved case, defined by $\Gamma=1$ (known as model A).

Even though the theoretical approach that will be presented here is quite general, our results will be applied, for the sake of clarity, to the particular Ginzburg-Landau form of the free energy $F$,

$$
F=\int d x\left[V(\phi)+\frac{D}{4 d}|\vec{\nabla} \phi|^{2}\right],
$$

where the local potential $V(\phi)$ is

$$
V(\phi)=-\frac{a}{2} \phi^{2}+\frac{1}{4} \phi^{4} .
$$

In the absence of noise sources, the behavior of this potential is the following: for $a \leqslant 0$ the homogeneous trivial solution $\phi=0$ is stable, whereas for $a>0$ that solution becomes unstable. This instability gives rise either to a phase transition towards an ordered (ferromagnetic) phase in the nonconserved case, or to a phase separation process in the conserved case.

The external noise will be taken to be coupled to the field according to

$$
g(\phi)=\phi,
$$

which corresponds to allow the control parameter $a$ in Eq. (5) to fluctuate in space and time. We will use the following Gaussian spatial correlation function:

$$
c\left(\left|\vec{x}-\vec{x}^{\prime}\right|\right)=\frac{1}{(\lambda \sqrt{2 \pi})^{d}} \exp \left(-\frac{\left|\vec{x}-\vec{x}^{\prime}\right|^{2}}{2 \lambda^{2}}\right),
$$

whose width $\lambda$ characterizes the correlation length of the noise. The normalization is such that in the limit $\lambda \rightarrow 0$ this correlation goes to a $\delta$ function and $\xi(\vec{x}, t)$ becomes a spatial white noise with intensity $\sigma^{2}$.

\section{MEAN-FIELD APPROACH FOR NONCONSERVED DYNAMICS}

We now review the main points of the mean-field approach in its application to nonconserved order-parameter systems (model $A$ ), in order to clarify the extension to conserved dynamics that will be presented in the next section. We begin by discretizing the field equation (1) with $\Gamma=1$ in a regular $d$-dimensional lattice of mesh size $\Delta x=1$ and lattice points $\vec{x}_{1}, \ldots, \vec{x}_{N}$,

$$
\frac{d \phi_{i}}{d t}=f\left(\phi_{i}\right)+\frac{D}{2 d} \sum_{j} \widetilde{D}_{i j} \phi_{j}+\eta_{i}(t)+g\left(\phi_{i}\right) \xi_{i}(t),
$$

where $\phi_{i} \equiv \phi\left(\vec{x}_{i}\right), f\left(\phi_{i}\right)=-V^{\prime}\left(\phi_{i}\right)$, and only one index is used to label the cells, independently of the dimension of the lattice. $\widetilde{D}_{i j}$ accounts for the discretized Laplacian operator

$$
\nabla^{2} \rightarrow \sum_{j} \widetilde{D}_{i j}=\sum_{j}\left(\delta_{\operatorname{nn}(i), j}-2 d \delta_{i, j}\right),
$$

where $n n(i)$ represents the set of all the sites which are nearest neighbors of site $i$.

The discrete noises $\eta_{i}(t)$ and $\xi_{i}(t)$ are still Gaussian with zero mean and their correlations are given by

$$
\left\langle\eta_{i}(t) \eta_{j}\left(t^{\prime}\right)\right\rangle=2 \varepsilon \delta_{i, j} \delta\left(t-t^{\prime}\right)
$$

and

$$
\left\langle\xi_{i}(t) \xi_{j}\left(t^{\prime}\right)\right\rangle=2 \sigma^{2} c_{|i-j|} \delta\left(t-t^{\prime}\right),
$$

where $c_{|i-j|}$ is a convenient discretization of the function $c\left(\left|\vec{x}-\vec{x}^{\prime}\right|\right)$ and specific values such as $c_{0}, c_{1}$ have to be computed numerically $[4,16]$ when needed. For the white-noise 
case $(\lambda=0)$ and with the mesh size chosen $\Delta x=1$ it is $c_{0}$ $=1, c_{1}=0$, whereas for large $\lambda, c_{0}$ scales roughly as $c_{0}$ $\propto \lambda^{-d}$.

The corresponding Fokker-Planck equation, in the Stratonovich interpretation, for the multivariate probability density $P\left(\phi_{1}, \phi_{2}, \ldots, t\right) \equiv P(\{\phi\}, t)$ is $[4]$

$$
\begin{aligned}
\frac{\partial P}{\partial t}= & -\sum_{i} \frac{\partial}{\partial \phi_{i}}\left[f\left(\phi_{i}\right)+\frac{D}{2 d} \sum_{j \in \mathrm{nn}(i)}\left(\phi_{j}-\phi_{i}\right)-\varepsilon \frac{\partial}{\partial \phi_{i}}\right. \\
& \left.-\sigma^{2} g\left(\phi_{i}\right) \sum_{j} c_{|i-j|} \frac{\partial}{\partial \phi_{j}} g\left(\phi_{j}\right)\right] P .
\end{aligned}
$$

In order to get the evolution equation for the single-site probability distribution $P\left(\phi_{i}, t\right)$, defined as

$$
P\left(\phi_{i}, t\right)=\int\left[\prod_{k \neq i} d \phi_{k}\right] P(\{\phi\}, t),
$$

we integrate Eq. (12) over all the variables except $\phi_{i}$. Vanishing of the probability for the field going to $\pm \infty$ leads to

$$
\int\left[\prod_{k \neq i} d \phi_{k}\right] \frac{\partial}{\partial \phi_{j}}\left[g\left(\phi_{j}\right) P(\{\phi\}, t)\right]=0, \quad j \neq i
$$

and using the standard definition of the conditional probability, one gets

$$
\begin{aligned}
& \sum_{j \in \mathrm{nn}(i)} \int\left[\prod_{k \neq i} d \phi_{k}\right] \phi_{j} P(\{\phi\}, t) \\
& \equiv\left[\sum_{j \in \mathrm{nn}(i)} \int d \phi_{j} \phi_{j} P\left(\phi_{j} \mid \phi_{i}, t\right)\right] P\left(\phi_{i}, t\right) \\
& \equiv 2 d\langle\phi(t)\rangle_{\phi_{i}} P\left(\phi_{i}, t\right)
\end{aligned}
$$

which defines $\langle\phi(t)\rangle_{\phi_{i}}$ as a nearest-neighbor conditional average. Thus we finally find that the one-point steady probability distribution follows the simpler but still exact equation,

$$
\begin{aligned}
\frac{\partial P\left(\phi_{i}, t\right)}{\partial t}= & -\frac{\partial}{\partial \phi_{i}}\left(f\left(\phi_{i}\right)+D\left[\langle\phi(t)\rangle_{\phi_{i}}-\phi_{i}\right]-\varepsilon \frac{\partial}{\partial \phi_{i}}\right. \\
& \left.-\sigma^{2} c_{0} g\left(\phi_{i}\right) \frac{\partial}{\partial \phi_{i}} g\left(\phi_{i}\right)\right) P\left(\phi_{i} t\right) .
\end{aligned}
$$

The mean-field approximation consists in assuming that the conditional average in the last equation is replaced by [26]

$$
\langle\phi(t)\rangle_{\phi_{i}}=\left\langle\phi_{i}(t)\right\rangle
$$

which is equivalent to doing directly the following assumption at the level of the Langevin Eq. (8):

$$
\frac{1}{2 d} \sum_{j} \widetilde{D}_{i j} \phi_{j}(t)=\left\langle\phi_{i}(t)\right\rangle-\phi_{i}(t) .
$$

Using this approximation, and imposing the condition of stationary probability distribution with no flux, we get that the single-site steady distribution satisfies

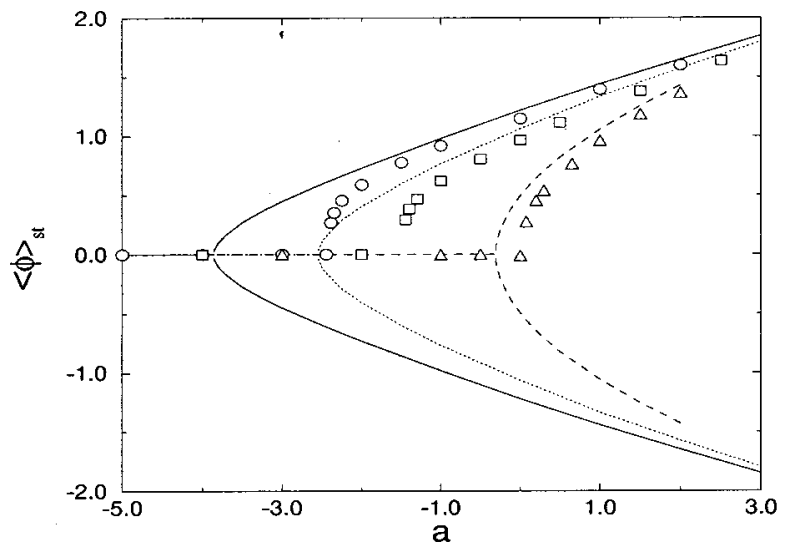

FIG. 1. Steady-state order parameter $\langle\phi\rangle_{\mathrm{st}}$ versus control parameter $a$ for model $A$. Lines are mean-field results and points correspond to numerical simulations of the complete model for a twodimensional square lattice with $64 \times 64$ cells, mesh size $\Delta x=1$, for system parameters $\lambda=0.0$ (circles and solid line), $\lambda=0.5$ (squares and dotted line), and $\lambda=1.5$ (triangles and dashed line). Other parameters are $D=3.7, \varepsilon=0.1$, and $\sigma^{2}=5.0$. All simulations in this paper use the same lattice parameters.

$$
\begin{aligned}
& {\left[f(\phi)+D\left(\langle\phi\rangle_{\mathrm{st}}-\phi\right)-\varepsilon \frac{\partial}{\partial \phi}-\sigma^{2} c_{0} g(\phi) \frac{\partial}{\partial \phi} g(\phi)\right] P_{\mathrm{st}}(\phi)} \\
& \quad=0
\end{aligned}
$$

where subscript $i$ has been dropped for simplicity.

The solution of the previous equation can be easily written down:

$$
\begin{aligned}
P_{\mathrm{st}}\left(\phi,\langle\phi\rangle_{\mathrm{st}}\right)= & N \exp \left(\int d \phi^{\prime} \frac{1}{\sigma^{2} c_{0} g^{2}\left(\phi^{\prime}\right)+\varepsilon}\right. \\
& \times\left[f\left(\phi^{\prime}\right)+D\left(\langle\phi\rangle_{\mathrm{st}}-\phi^{\prime}\right)\right. \\
& \left.\left.-\sigma^{2} c_{0} g\left(\phi^{\prime}\right) g^{\prime}\left(\phi^{\prime}\right)\right]\right),
\end{aligned}
$$

where $N$ is an appropriate normalization constant. The above solution is only formal, because $\langle\phi\rangle_{\text {st }}$ depends on the probability distribution itself. However, both $P_{\mathrm{st}}$ and $\langle\phi\rangle_{\mathrm{st}}$ can be determined by means of the following self-consistency relation, which is a signature of the mean-field approach,

$$
\langle\phi\rangle_{\mathrm{st}}=\int_{-\infty}^{\infty} \phi P_{\mathrm{st}}\left(\phi,\langle\phi\rangle_{\mathrm{st}}\right) d \phi .
$$

We now apply our results to the particular model defined by Eqs. (4)-(7). The solution of the self-consistency equation (21) in this case is plotted in Fig. 1 versus the control parameter $a$ for three different values of the noise correlation length $\lambda$. Note that the existence of multiplicative noise shifts the critical point towards negative values of $a$, which indicates the ordering character of the external noise. This noise-induced phase transition has been substantially studied in the past in the case of white external noise $[8,17,22]$. Figure 1 also displays the results obtained by numerical simulations of the complete model (8) for a two-dimensional square lattice and using the mean-field results as the initial 
conditions. It can be seen that mean-field results give the correct qualitative behavior of the system, and are also quantitatively right far from the critical point. The agreement between mean-field predictions and simulations close to the critical point improves when the correlation length $\lambda$ increases.

Notice that, in this mean-field approximation, the only effect of the finite correlation length $\lambda$ of the noise shows up in the value $c_{0}<1$ [see Eq. (20)] of the correlation function at zero distance. As mentioned before, this value decreases with increasing $\lambda$. In other words, for nonconserved dynamics the disordering effect of the spatial correlation of multiplicative noise in the mean-field approximation arises only through a decrease of the effective noise intensity.

\section{MEAN-FIELD APPROACH FOR CONSERVED DYNAMICS}

The mean-field approach discussed above cannot be straightforwardly extended to deal with conserved-orderparameters systems, because in these cases the mean field $\langle\phi\rangle$ is constant in time, depending only on the initial conditions and not on the control parameter $a$. We now introduce a generalized mean-field approximation that overcomes such a restriction. The main ideas underlying this extension will be first presented in the deterministic model $B$.

\section{A. Deterministic dynamics}

In the absence of all noise sources, model $B$ takes the form

$$
\frac{\partial \phi(\vec{x}, t)}{\partial t}=\nabla^{2} \frac{\delta F}{\delta \phi}
$$

This model evolves in time under the following restriction:

$$
\frac{1}{V} \int d \vec{x} \phi(\vec{x}, t)=\phi_{0},
$$

where $\phi_{0}$ is fixed by the initial conditions. The phenomenology of this model is well known [25]: there is a transition point $a_{T}\left(\phi_{0}\right)$, such that for $a<a_{T}\left(\phi_{0}\right)$ the homogeneous state $\phi=\phi_{0}$ is stable, whereas for $a>a_{T}\left(\phi_{0}\right)$ the system separates in two bulk phases, $\phi_{1}$ and $\phi_{2}$, fulfilling that the spatial average of $\phi$ is also equal to $\phi_{0}$. The transition from a homogeneous state to a two-phase state is critical (i.e., of second order) for $\phi_{0}=0$, so that $a_{T}\left(\phi_{0}=0\right) \equiv a_{c}$.

In order to determine both $a_{T}\left(\phi_{0}\right)$ and $a_{c}$, we look for the steady-state solutions of Eq. (22). These solutions fulfill the Laplace equation $\nabla^{2}(\delta F / \delta \phi)=0$. The analytical and bounded solution is $\delta F / \delta \phi=h$, where $h$ is a constant. Therefore the steady states of model $B$ can be interpreted as the minima of an effective potential $F_{\text {eff }}=F-h \int d \vec{x} \phi$, and coincide with the steady states of model $A$ with an external control field $h$. Following Ref. [27], we call $h$ the constant effective field of the system. For equilibrium systems, $h$ is merely the chemical potential. Moreover, $h$ is not an arbitrary constant, and it has to be determined by imposing the conservation law, Eq. (23). Substitution of the GinzburgLandau form in the discretized version of $h=\delta F / \delta \phi$ leads to

$$
h=-a \phi_{i}+\phi_{i}^{3}-\frac{D}{2 d} \sum_{j} \widetilde{D}_{i j} \phi_{j}
$$

We now need to consider separately the subthreshold and superthreshold situations.

(i) In the subthreshold (homogeneous) case, the condition $\phi_{i}=\phi_{0}, \forall i$, has to be verified, and therefore Eq. (24) reduces to

$$
h=-a \phi_{0}+\phi_{0}^{3} .
$$

Hence the value of $h$ does depend on the initial condition in the subthreshold situation.

(ii) Above the transition point (not yet determined), the steady state of the system is not globally homogeneous, since the field separates in two bulk phases with values $\phi_{1}$ and $\phi_{2}$, respectively. The fraction $x$ of the system in phase $\phi_{1}$ is given by the lever rule: $x \phi_{1}+(1-x) \phi_{2}=\phi_{0}$. For a general free energy, a Maxwell-type construction would give us the value of $h$. In the case of a locally symmetric free energy [such as the one defined in Eqs. (4) and (5)], a simpler argument can be used: each phase has to satisfy Eq. (24) with $\phi_{i}$ equal to the field value of the corresponding phase, either $\phi_{1}$ or $\phi_{2}$, and, since by the symmetry of the free energy, these two quantities verify $\phi_{1}=-\phi_{2}$, and $h$ must be zero. Consequently, we get

$$
\phi_{1,2}= \pm \sqrt{a},
$$

which are the solutions of the deterministic model $A$ for a value of the external control field $h=0$.

Just at the transition point, there is a unique phase $\phi$ $=\phi_{0}$ and $h$ is identically zero. Thus the transition line (also called in this context the coexistence line) is given by

$$
a_{T}\left(\phi_{0}\right)=\phi_{0}^{2} .
$$

We also note that for $\phi_{0}=0$, the critical point is obtained: $a_{T}=a_{c}=0$.

We will now show that the concept of the constant effective field can be used to generalize the mean-field approximation to conserved systems with noise.

\section{B. Noise-induced phase separation}

We now add stochastic sources to model (22), in the form of both an internal additive noise and an external multiplicative one. The resulting model is represented by Eqs. (1)-(3) with $\Gamma=-\nabla^{2}$. The discretized version of this model is

$$
\frac{d \phi_{i}}{d t}=-\sum_{j} \widetilde{D}_{i j}\left(f\left(\phi_{j}\right)+\frac{D}{2 d} \sum_{k} \widetilde{D}_{j k} \phi_{k}+g\left(\phi_{j}\right) \xi_{j}\right)+\eta_{i}(t),
$$

with $f\left(\phi_{j}\right)=-V^{\prime}\left(\phi_{j}\right)$, as before. The correlation of the additive noise is now

$$
\left\langle\eta_{i}(t) \eta_{j}\left(t^{\prime}\right)\right\rangle=-2 \varepsilon \widetilde{D}_{i j} \delta\left(t-t^{\prime}\right),
$$

and that of the multiplicative noise was already introduced in Eq. (11). The corresponding Fokker-Planck equation, in the Stratonovich interpretation, for the multivariate probability density $P(\{\phi\}, t)$ is in this case 


$$
\begin{aligned}
\frac{\partial P}{\partial t}= & \sum_{i, j} \frac{\partial}{\partial \phi_{i}} \widetilde{D}_{i j}\left(f\left(\phi_{j}\right)+\frac{D}{2 d} \sum_{k} \widetilde{D}_{j k} \phi_{k}-\varepsilon \frac{\partial}{\partial \phi_{j}}\right. \\
& \left.+\sigma^{2} g\left(\phi_{j}\right) \sum_{r, s} \frac{\partial}{\partial \phi_{s}} \widetilde{D}_{s r} c_{|j-r|} g\left(\phi_{r}\right)\right) P .
\end{aligned}
$$

As done in the nonconserved case, we now integrate Eq. (30) over all the variables except $\phi_{i}$, in order to get the evolution equation of the single-site probability distribution $P\left(\phi_{i}, t\right)$ [see Eq. (13)],

$$
\frac{\partial P\left(\phi_{i}, t\right)}{\partial t}=\frac{\partial}{\partial \phi_{i}} \sum_{j} \widetilde{D}_{i j}\left\langle M_{j}\right\rangle_{\phi_{i}} P\left(\phi_{i}, t\right),
$$

where

$$
\begin{aligned}
M_{j}= & f\left(\phi_{j}\right)+\frac{D}{2 d} \sum_{k} \widetilde{D}_{j k} \phi_{k}-\varepsilon \frac{\partial}{\partial \phi_{j}} \\
& +\sigma^{2} g\left(\phi_{j}\right) \sum_{r, s} \frac{\partial}{\partial \phi_{s}} \widetilde{D}_{s r} c{ }_{|j-r|} g\left(\phi_{r}\right) .
\end{aligned}
$$

If we impose the condition of stationarity probability distribution with no flux, $\left\langle M_{j}\right\rangle_{\phi_{i}}$ must satisfy

$$
\sum_{j} \widetilde{D}_{i j}\left\langle M_{j}\right\rangle_{\phi_{i}} P_{\mathrm{st}}\left(\phi_{i}\right)=0
$$

In the deterministic conserved case it has been shown that the solution of this equation is the constant effective field, $\left\langle M_{j}\right\rangle_{\phi_{i}}=-h$. We can now take $j=i$ and perform the conditional average of $M_{i}$. If we consider the expression analogous to Eq. (14) for the multiplicative noise term and make the standard mean-field approximation (17), we arrive at

$$
\begin{aligned}
-h P_{\mathrm{st}}(\phi)= & \left(f(\phi)+D\left(\langle\phi\rangle_{\mathrm{st}}-\phi\right)-\varepsilon \frac{\partial}{\partial \phi}+2 d \sigma^{2} g(\phi)\right. \\
& \left.\times\left[c_{1} g\left(\langle\phi\rangle_{\mathrm{st}}\right) \frac{\partial}{\partial \phi}-c_{0} \frac{\partial}{\partial \phi} g(\phi)\right]\right) P_{\mathrm{st}}(\phi),
\end{aligned}
$$

where subscripts have been dropped again for simplicity. In the derivation of this equation, a generalization of the meanfield approximation for the nearest-neighbor conditional average of function $g(\phi)$ has been applied, namely

$$
\langle g(\phi)\rangle_{\phi_{i}}=g\left(\left\langle\phi_{i}\right\rangle\right)
$$

In principle this is an uncontrolled approximation whose validity needs to be assessed by the numerical simulations, which will be presented in what follows.

The solution of Eq. (34) yields the following stationary probability distribution:

$$
\begin{aligned}
P_{\mathrm{st}}\left(\phi,\langle\phi\rangle_{\mathrm{st}}, h\right)= & N \exp \left(\int \frac{d \phi^{\prime}}{2 d \sigma^{2} g\left(\phi^{\prime}\right)\left[c_{0} g\left(\phi^{\prime}\right)-c_{1} g\left(\langle\phi\rangle_{\mathrm{st}}\right)\right]+\varepsilon}\right. \\
& \left.\times\left[f\left(\phi^{\prime}\right)+D\left(\langle\phi\rangle_{\mathrm{st}}-\phi^{\prime}\right)-2 d \sigma^{2} c_{0} g\left(\phi^{\prime}\right) g^{\prime}\left(\phi^{\prime}\right)+h\right]\right),
\end{aligned}
$$

where $h$ and $\langle\phi\rangle_{\text {st }}$ are parameters to be determined self-consistently.

We now particularize the result obtained above to the Ginzburg-Landau model defined by Eqs. (4)-(7). In this case, the stationary single-site probability distribution is

$$
P_{\mathrm{st}}\left(\phi,\langle\phi\rangle_{\mathrm{st}}, h\right)=N \exp \left(\int \frac{\left(a-D-2 d \sigma^{2} c_{0}\right) \phi^{\prime}-\phi^{\prime 3}+D\langle\phi\rangle_{\mathrm{st}}+h}{2 d \sigma^{2}\left(c_{0} \phi^{\prime 2}-c_{1}\langle\phi\rangle_{\mathrm{st}} \phi^{\prime}\right)+\varepsilon} d \phi^{\prime}\right) .
$$

We now need to determine the unknown constants $h$ and $\langle\phi\rangle_{\text {st }}$. Similarly to the deterministic case studied above, we consider separately the subthreshold and superthreshold situations. We recall at this point that the mean-field approach presented above is local, and leads to an expression for the probability distribution of the field at a given site of the lattice as a function of $h$ and of the mean field $\langle\phi\rangle_{\text {st }}$ in the neighborhood of the given cell. In the homogeneous case $\left(a<a_{T}\right)$, this mean field is the same everywhere, and it is equal to $\phi_{0}$. Hence only $h$ is left to be evaluated, which can be done by means of the generalized self-consistency relation

$$
\langle\phi\rangle_{\mathrm{st}}=\int_{-\infty}^{\infty} \phi P_{\mathrm{st}}\left(\phi,\langle\phi\rangle_{\mathrm{st}}, h\right) d \phi
$$

with $\langle\phi\rangle_{\mathrm{st}}=\phi_{0}$.

For $a>a_{T}$, the system has two phases, and thus there are two different local mean values, corresponding to each of the bulk phases $\left(\langle\phi\rangle_{1}\right.$ and $\left.\langle\phi\rangle_{2}\right)$. Because of the symmetry of $F$ and $g$, these values must satisfy $\langle\phi\rangle_{1}=-\langle\phi\rangle_{2}$. Therefore, since $h$ needs to be the same for the two phases, and given the form of the numerator in Eq. (37), $h$ must be zero in this ordered state. Hence only the values of the local (symmetrical) mean fields $\langle\phi\rangle_{1}$ and $\langle\phi\rangle_{2}$ need to be determined for $a>a_{T}$. This can be done as in the case of model $A$, solving the self-consistency relation (21) using the steady probability given in Eq. (37) with $h=0$. For nonsymmetric functions $F(\phi)$ and $g(\phi)$, a possible extension of the Maxwell rule is to choose $h$ in such a way that the two solutions of Eq. (38) 


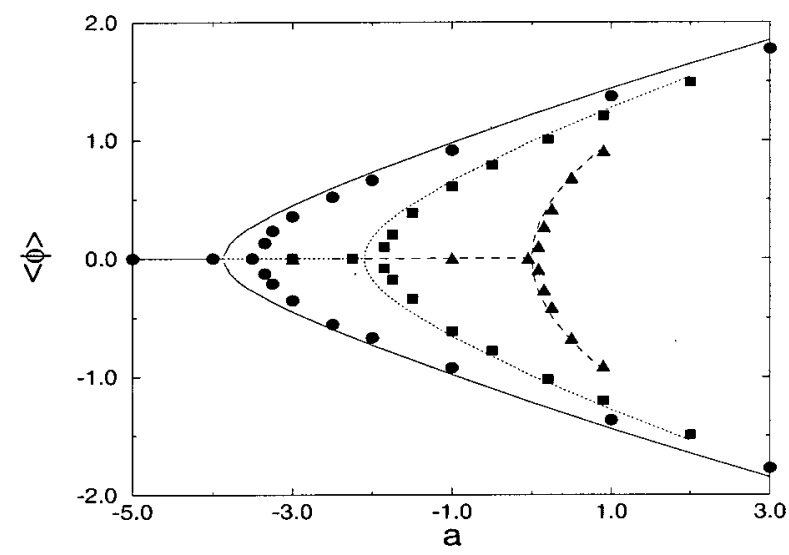

FIG. 2. Steady-state bulk order parameter $\langle\phi\rangle$ versus control parameter $a$ for model $B$. Lines are mean-field results and points correspond to numerical simulations for $\lambda=0.0$ (circles and solid line), $\lambda=0.5$ (squares and dotted line) and $\lambda=1.5$ (triangles and dashed line). Other parameters are $D=3.7, \varepsilon=0.1$, and $\sigma^{2}=1.25$.

have the same value for the stationary probability $P_{\mathrm{st}}\left(\langle\phi\rangle_{\mathrm{st}},\langle\phi\rangle_{\mathrm{st}}, h\right)$.

The bifurcation diagram resulting from the application of the self-consistency relations is plotted in Fig. 2 for three different values of the multiplicative-noise correlation length. Numerical simulation results of the complete model (28) are also shown. Mean-field results have been used as the initial conditions of the numerical simulations letting each phase evolve until its stationary value. The effects of the intensity and correlation length of the multiplicative noise are qualitatively the same as in model $A$ : the noise-induced shift of the transition point, in the direction of enhancing order in the system, increases with noise intensity and decreases with correlation length.

Figure 3 shows the values of the constant effective field $h$ obtained numerically by imposing the self-consistency relation (38) until $h$ vanishes, for a nonzero initial concentration $\phi_{0}=0.2$. The corresponding value of the control parameter $a$ at which $h$ first becomes zero is the transition point $a_{T}$. Results have been plotted for the deterministic case [which can be calculated analytically, see Eq. (25)], the case with

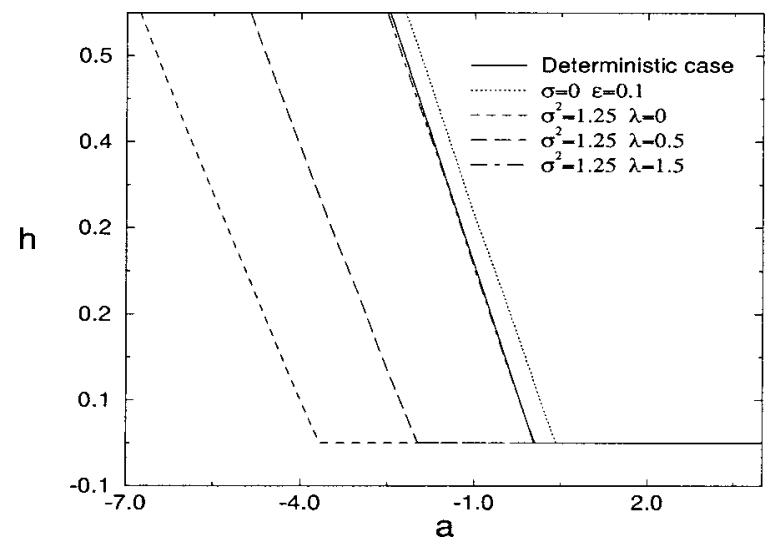

FIG. 3. Constant effective field $h$ as a function of the control parameter $a$, as obtained from mean-field theory, for $D=3.7$ and $\phi_{0}=0.2$. All the cases with multiplicative noise have also additive noise with $\epsilon=0.1$. just additive noise, and three cases with also multiplicative noise for different correlation lengths, corresponding to the situations shown in Fig. 2. The noise-induced shift of the transition point and the influence of the noise correlation length as well as the disordering role of the additive noise (reflected in the shift of the transition point towards the right when only additive noise is considered — dotted line) can be clearly seen.

A comment on the comparative influence of multiplicative noise on conserved and nonconserved dynamics is worth making at this point. We note that, in the ordered state ( $h$ $=0$ ), the single-site probability distribution of the conserved model (36) in the presence of white multiplicative noise $\left(c_{0}=1\right.$ and $\left.c_{1}=0\right)$ reduces to

$$
\begin{aligned}
P_{\mathrm{st}}\left(\phi,\langle\phi\rangle_{\mathrm{st}}\right)= & N\left(\operatorname { e x p } \int d \phi ^ { \prime } \frac { 1 } { 2 d \sigma ^ { 2 } g ^ { 2 } ( \phi ^ { \prime } ) + \varepsilon } \left[f\left(\phi^{\prime}\right)\right.\right. \\
& \left.\left.+D\left(\langle\phi\rangle_{\mathrm{st}}-\phi^{\prime}\right)-2 d \sigma^{2} g\left(\phi^{\prime}\right) g^{\prime}\left(\phi^{\prime}\right)\right]\right),
\end{aligned}
$$

which should be compared with the corresponding expression (20) for the nonconserved case with $c_{0}=1$. One can easily see that multiplicative noise has a stronger effect on the conserved model than on the nonconserved one, since in the former case the noise intensity is multiplied by a factor $2 d$. In the particular case in which the two noise intensities of the nonconserved $(A)$ and conserved $(B)$ models are related by

$$
\sigma_{A}^{2}=2 d \sigma_{B}^{2}
$$

the two models are equivalent above the transition point. However, this equivalence disappears in the case of colored multiplicative noise, because of the term $2 d \sigma^{2} c_{1} g\left(\phi^{\prime}\right) g\left(\langle\phi\rangle_{\mathrm{st}}\right)$ appearing in Eq. (36). This different dependence indicates that spatial correlation of the noise is more relevant for the conserved model than for the nonconserved one, where the correlation length of the noise produces only a shift of the transition point [16]. A comparison between the results of models $A$ and $B$ is shown in Fig. 4, for both $\lambda=0$ and $\lambda \neq 0$. Noise intensities have been chosen here to verify expression (40), so that in the white-noise case, mean-field results coincide for the two models. Mean-field results are in better agreement with simulations in the case of model $B$.

Finally, we now address the issue of whether a reentrant noise-induced phase transition towards disorder arises in the conserved model $B$. Previous works have shown the existence of such a transition for nonconserved models [7,8]. This means that for fixed values of $a$ and $D$, when increasing the multiplicative noise intensity, the system goes first through a phase transition from disorder to order (NIOT) and then, for higher values of the noise, it experiments another transition back to disorder (NIDT). These two transitions can only be found when increasing $\sigma^{2}$, instead of $a$ or $D$.

Mean-field theory predicts the existence of reentrant transitions also for model $B$, as shown in Fig. 5. This figure shows the behavior of the mean field $\langle\phi\rangle_{\text {st }}$ versus multipli- 


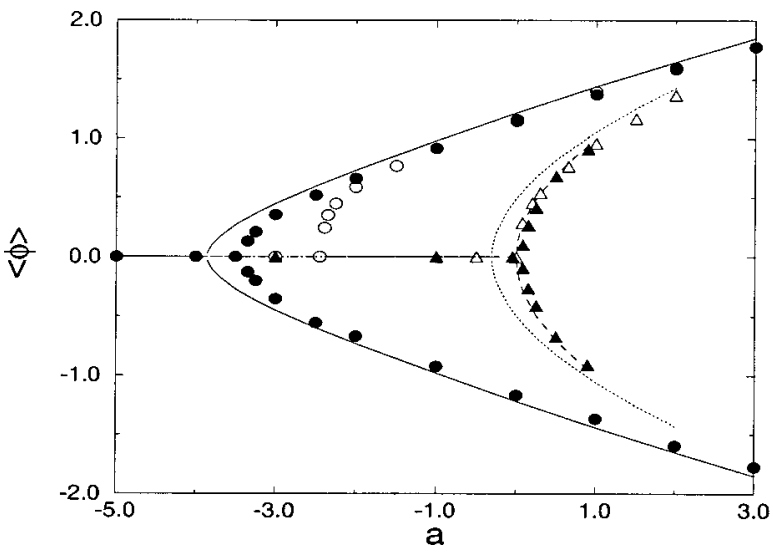

FIG. 4. Steady-state bulk order parameters $\langle\phi\rangle$ versus control parameter $a$ for model $A$ and $B$ with additive white and multiplicative colored noises for different correlation lengths. Lines are meanfield results for model $A$ with $\lambda=0.0$ (solid line) and $\lambda=1.5$ (dotted line) and for model $B$ with $\lambda=0.0$ (solid line) and $\lambda=1.5$ (dashed line). Points correspond to numerical simulations of model $A$ (empty symbols) and model $B$ (full symbols). Of these, circles correspond to white multiplicative noise and triangles to $\lambda=1.5$. Other parameters are $D=3.7, \varepsilon=0.1, \sigma_{A}^{2}=5$, and $\sigma_{B}^{2}=1.25$.

cative noise intensity with two different correlation lengths ( $\lambda=0$ and $\lambda=0.5$ ) for models $A$ and $B$. Again, the noise intensities for the two models have been chosen to verify Eq. (40), so that the $\lambda=0$ result is identical in the two cases. However, the effect of the correlation length is different for the two models: whereas for model $A \lambda$ retards both the NIOT and the NIDT, for model $B$ the NIOT is retarded, but the NIDT is advanced. This is an indication of the nontrivial influence of the noise correlation length in the conserved case.

\section{STRONG-COUPLING LIMIT}

In the limit of strong coupling $D \rightarrow \infty$, the predictions of mean-field theory can be evaluated analytically and should agree with the results given by a standard linear stability

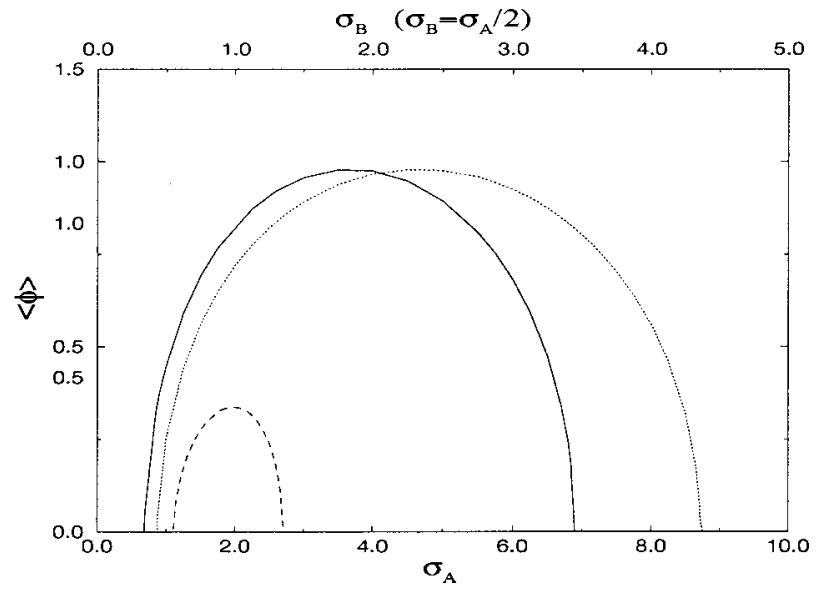

FIG. 5. Mean field steady-state bulk order parameters $\langle\phi\rangle$ versus multiplicative noise intensity for models $A$ with $\lambda=0.0$ (solid line) and $\lambda=0.5$ (dotted line) and for model $B$ with $\lambda=0.0$ (solid line) and $\lambda=0.5$ (dashed line). Parameters are $\epsilon=1, a=0.75$, and $D=2.66$. analysis of the model. In order to verify this agreement, we will now compute this limit for the mean-field results obtained so far, for both models $A$ and $B$.

\section{A. Model $A$}

In the mean-field approximation and in the limit $D \rightarrow \infty$, the stationary probability distribution $P_{\mathrm{st}}\left(\phi,\langle\phi\rangle_{\mathrm{st}}\right)$ (20) becomes

$$
P_{\mathrm{st}}\left(\phi,\langle\phi\rangle_{\mathrm{st}}\right)=\delta\left(\phi-\langle\phi\rangle_{\mathrm{st}}\right),
$$

as can be easily seen by means of a steepest-descent calculation. This expression verifies trivially the self-consistency relation (21), which can thus no longer be used to determine $\langle\phi\rangle_{\text {st }}$. In order to do that, we now integrate Eq. (19) with respect to $\phi$, and obtain

$$
\langle f(\phi)\rangle_{\mathrm{st}}+\sigma^{2} c_{0}\left\langle g^{\prime}(\phi) g(\phi)\right\rangle_{\mathrm{st}}=0 .
$$

For $D \rightarrow \infty$, these averages are evaluated trivially using expression (41), and Eq. (42) becomes

$$
f\left(\langle\phi\rangle_{\mathrm{st}}\right)+\sigma^{2} c_{0} g^{\prime}\left(\langle\phi\rangle_{\mathrm{st}}\right) g\left(\langle\phi\rangle_{\mathrm{st}}\right)=0,
$$

from which $\langle\phi\rangle_{\text {st }}$ can be found. For model $A$ and in the case defined by Eqs. (4)-(7), the solutions of this equation are either

$$
\langle\phi\rangle_{\mathrm{st}}=0
$$

or

$$
\langle\phi\rangle_{1,2}= \pm \sqrt{a+\sigma^{2} c_{0}} \text {. }
$$

This second set of solutions can only exist for $a>-\sigma^{2} c_{0}$. Hence, the critical point is given in this case by

$$
a_{c}=-\sigma^{2} c_{0},
$$

in such a way that the ordered state appears for $a>a_{c}$. The shift of the critical point increases the ordered region, due to the effective multiplicative noise intensity $\sigma^{2} c_{0}$. This shift, as seen in the previous sections, increases with increasing noise intensity and decreases for increasing correlation lengths. This result coincides with the one given by a linear stability analysis of the homogeneous state [4,16,17], as expected. However, in contrast with the linear stability analysis, this calculation can be extended to other situations and models not necessarily controlled by the linear term.

\section{B. Model $\boldsymbol{B}$}

In this case, the stationary probability distribution (36) given by the mean-field approach for each phase and for $D$ $\rightarrow \infty$ is also Eq. (41) as can be seen using the steepestdescent method, as before. Following the procedure described above for model $A$, we formally integrate now Eq. (34) to obtain an equation for $h$,

$$
\begin{aligned}
h= & -\langle f(\phi)\rangle_{\mathrm{st}}+2 d \sigma^{2}\left[c_{1} g\left(\langle\phi\rangle_{\mathrm{st}}\right)\left\langle g^{\prime}(\phi)\right\rangle_{\mathrm{st}}\right. \\
& \left.-c_{0}\left\langle g(\phi) g^{\prime}(\phi)\right\rangle_{\mathrm{st}}\right] .
\end{aligned}
$$




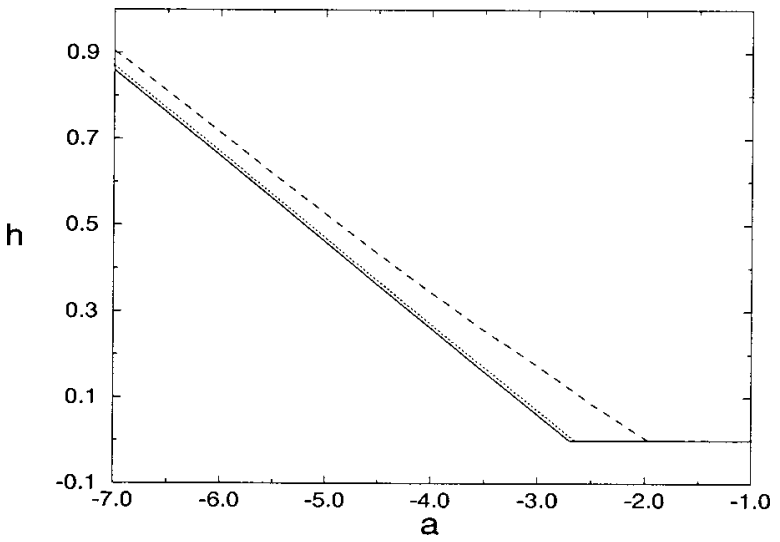

FIG. 6. Constant effective field $h$ versus control parameter $a$ for $D \rightarrow \infty$ (solid line) as given by Eq. (48), and for $D=3.7$ (dashed line) and $D=20$ (dotted line) coming from the mean-field approach described in Sec. IV B. Other parameters are $\phi_{0}=0.2, \epsilon=0.1, \sigma^{2}$ $=1.25$, and $\lambda=0.5$.

At the limit $D \rightarrow \infty$, the averages appearing in the preceding expression are calculated using the stationary probability distribution obtained above, leading to

$$
h=-f\left(\langle\phi\rangle_{\mathrm{st}}\right)+2 d \sigma^{2}\left(c_{1}-c_{0}\right) g^{\prime}\left(\langle\phi\rangle_{\mathrm{st}}\right) g\left(\langle\phi\rangle_{\mathrm{st}}\right) .
$$

In the case $a<a_{T}$, the field is homogeneous and we can replace in the above expression $\langle\phi\rangle_{\mathrm{st}}=\phi_{0}$. Thus this equation gives us the value of $h$ in this case as a function of the initial condition. The results for model $B$ and in the case defined by Eqs. (4)-(7) are plotted in Fig. 6 versus the control parameter $a$, along with the values of $h$ given by meanfield theory for finite but large $D$, obtained numerically in the preceding section. We can see that these mean-field results approach Eq. (48) as $D$ increases, as it should be. The shift of the transition point increases for increasing coupling strength, as can be seen from Fig. 6 .

We now turn to the case $a>a_{T}$, where $h=0$. Now Eq. (48) can be solved for $\langle\phi\rangle_{\text {st }}$, which gives the values of the two bulk phases,

$$
\langle\phi\rangle_{1,2}= \pm \sqrt{a+2 d \sigma^{2}\left(c_{0}-c_{1}\right)},
$$

again for the particular model (4)-(7). The transition line is determined by setting $\langle\phi\rangle_{1}=\phi_{0}$ in the preceding expression, which leads to

$$
a_{T}=\phi_{0}^{2}-2 d \sigma^{2}\left(c_{0}-c_{1}\right),
$$

and the critical point (for $\phi_{0}=0$ ) is then

$$
a_{c}=-2 d \sigma^{2}\left(c_{0}-c_{1}\right) .
$$

This result coincides with that coming from linear stability analysis [16]. As in model $A$ and in the previous sections, the shift is in the direction of increasing the ordered region. Due to the factor $2 d$, this shift is larger than the one produced in model $A$ for the same noise intensities. Contrary to model $A$ in the colored case, the shift does not depend only on the effective multiplicative noise intensity $\sigma^{2} c_{0}$ but also on the noise correlation between first neighbors $c_{1}$, which indicates the nontrivial influence of the spatial correlation of the noise on conserved dynamics, as opposed to nonconserved dynamics where this influence disappears in the mean-field approach.

\section{CONCLUSIONS}

Mean-field theory has been previously applied to nonconserved models with additive and multiplicative white noises $[7,8,22]$. Here we have applied it in the case of spatially correlated multiplicative noise. Our mean-field results and numerical simulations of the complete model in two dimensions indicate the decrease of the ordering role of multiplicative noise when its correlation length increases.

We have also extended mean-field theory to deal with conserved models by using the concept of a constant effective field. As in the case of nonconserved systems, we have found that additive noise has a disordering role, whereas multiplicative noise has an ordering one. The latter increases for increasing multiplicative noise intensity and for decreasing noise correlation length. However, the quantitative effects of multiplicative noise are different in each model; the transition to order occurs earlier for model $B$ than for model $A$. Moreover, mean-field calculations show that the correlation length of multiplicative noise has nontrivial effects in the conserved case, while for model $A$ it just decreases the effective noise intensity. Numerical simulations of the complete conserved model in two dimensions are in good agreement with mean-field predictions.

Previous works on model $A$ with additive and multiplicative white noises have shown the presence of NIOTs and NIDTs. We have seen that, at least in the mean-field approach, these transitions appear for higher values of the noise intensity when multiplicative noise is spatially correlated. This is explained by the fact that the effective noise intensity decreases. Model $B$ has also been found to go first through a NIOT and after through a NIDT when the multiplicative noise intensity is increased. As in model $A$, the NIOT is retarded when the correlation length of multiplicative noise increases. However, contrary to what happens in model $A$, the NIDT is advanced, which shows clearly different effects of noise correlation length upon conserved and nonconserved models.

Finally, in the strong-coupling limit we have found analytical expressions for the critical-point shift and the steadystate bulk order parameter for both models $A$ and $B$ with additive white and multiplicative colored noises. These results coincide with previously reported predictions coming from linear stability analysis [16].

\section{ACKNOWLEDGMENTS}

This work has been supported by the Dirección General de Investigación Científica y Técnica (Spain), under projects PB97-0141-C02-01 PB94-1167, and PB96-0241. J.G.O. also acknowledges the Alexander von Humboldt-Stiftung (Germany) for financial support, and thanks the group of Professor Lutz Schimansky-Geier in the Humboldt Universität zu Berlin for their hospitality. 
[1] W. Horsthemke and R. Lefever, Noise-Induced Transitions (Springer, Berlin, 1984).

[2] L. Gammaitoni, P. Hänggi, P. Jung, and F. Marchesoni, Rev. Mod. Phys. 70, 223 (1998)

[3] P. Hänggi and R. Bartussek, in Nonlinear Physics of Complex Systems, edited by J. Parisi, S.C. Müller, and W. Zimmermann (Springer, Berlin, 1996).

[4] J. García-Ojalvo and J.M. Sancho, Noise in Spatially Extended Systems (Springer, New York, 1999).

[5] J. García-Ojalvo, A. Hernández-Machado, and J.M. Sancho, Phys. Rev. Lett. 71, 1542 (1993).

[6] J.M.R. Parrondo, C. Van den Broeck, J. Buceta, and F.J. de la Rubia, Physica A 224, 153 (1996).

[7] C. Van den Broeck, J.M.R. Parrondo, and R. Toral, Phys. Rev. Lett. 73, 3395 (1994); C. Van den Broeck, J.M.R. Parrondo, R. Toral, and R. Kawai, Phys. Rev. E 55, 4084 (1997).

[8] J. García-Ojalvo, J.M.R. Parrondo, J.M. Sancho, and C. Van den Broeck, Phys. Rev. E 54, 6918 (1996).

[9] S. Mangioni, R. Deza, H. Wio, and R. Toral, Phys. Rev. Lett. 79, 2389 (1997)

[10] P. Jung and G. Mayer-Kress, Phys. Rev. Lett. 74, 2134 (1995).

[11] F. Marchesoni, L. Gammaitoni, and A.R. Bulsara, Phys. Rev. Lett. 76, 2609 (1996).

[12] M.A. Santos and J.M. Sancho, Phys. Rev. E 59, 98 (1999).

[13] S. Kádár, J. Wang, and K. Showalter, Nature (London) 391, 770 (1998); P. Jung, A. Cornell-Bell, F. Moss, S. Kádár, J. Wang, and K. Showalter, Chaos 8, 567 (1998); J. Wang, S. Kádár, P. Jung, and K. Showalter, Phys. Rev. Lett. 82, 855 (1999).

[14] R.J. Deissler, J. Stat. Phys. 54, 1459 (1989).

[15] M. Santagiustina, P. Colet, M. San Miguel, and D. Walgraef,
Phys. Rev. Lett. 79, 3633 (1997); Phys. Rev. E 58, 3843 (1998).

[16] J. García-Ojalvo, A.M. Lacasta, J.M. Sancho, and R. Toral, Europhys. Lett. 42, 125 (1998).

[17] A. Becker, and L. Kramer, Phys. Rev. Lett. 73, 955 (1994); Physica D 90, 408 (1995).

[18] G. Grinstein, M.A. Muñoz, and Y. Tu, Phys. Rev. Lett. 76, 4376 (1996).

[19] J.M. Sancho, J. García-Ojalvo, and H. Guo, Physica D 113, 331 (1998).

[20] Y. Tu, G. Grinstein, and M.A. Muñoz, Phys. Rev. Lett. 78, 274 (1997).

[21] W. Genovese, M.A. Muñoz, and J.M. Sancho, Phys. Rev. E 57, R2495 (1998).

[22] C. Van den Broeck, J.M.R. Parrondo, J. Armero, and A. Hernández-Machado, Phys. Rev. E 49, 2639 (1994).

[23] P.S. Landa, A.A. Zaikin, and L. Schimansky-Geier, Chaos Solitons Fractals 9, 1367 (1998); A.A. Zaikin and L. Schimansky-Geier, Phys. Rev. E 58, 4355 (1998).

[24] Throughout this paper we follow the Stratonovich interpretation. In the Ito interpretation, where the homogeneous state is linearly stable, the noise-induced transitions reported in this paper will not appear.

[25] J.D. Gunton, M. San Miguel, and P.S. Sahni, in Phase Transitions and Critical Phenomena, edited by C. Domb and J.L. Lebowitz (Academic Press, New York, 1983), Vol. 8.

[26] In this assumption local correlations are discarded. A secondorder correction can be implemented as described in [7].

[27] M.A. Muñoz, U. Marini, and R. Cafiero, Europhys. Lett. 43, 552 (1998). 